\title{
Cutaneous Basal Cell Carcinoma Arising in Odontogenic Cutaneous Fistula
}

\author{
Nam Gyun Kim', \\ Jun Oh $\mathrm{Kim}^{1}$, \\ Young Ji Park ${ }^{1}$, \\ Jun Sik Kim ${ }^{1}$, \\ Yoon Jung Lee ${ }^{2}$, \\ Kyung Suk Lee ${ }^{1}$ \\ ${ }^{1}$ Department of Plastic and Reconstructive \\ Surgery, Institute of Health Sciences, College \\ of Medicine and Hospital, Gyeongsang Na- \\ tional University, Jinju; \\ ${ }^{2}$ Department of Plastic and Reconstructive \\ Surgery, Gyeongsang National University \\ Changwon Hospital, Changwon, Korea
}

No potential conflict of interest relevant to this article was reported.

\begin{abstract}
An odontogenic cutaneous fistula is a pathological communication between the outer skin surface of the face and the oral cavity. Facial cutaneous fistula is a complication of odontogenic infection that is often misdiagnosed with skin infection. We report a rare case, which was diagnosed as basal cell carcinoma based on the biopsy of skin lesions in the patient who had been diagnosed with odontogenic cutaneous fistula. A 64-year-old male patient presented with a cutaneous odontogenic fistula. The patient had undergone surgical extraction of fistula tract and loose tooth before dermatology or plastic surgery consultation. With the biopsy and computed tomography, it was confirmed that fistula and basal cell carcinoma. However, the connection between the fistula and skin cancer was not clear. Positron emission tomography-computed tomography scan was performed and was not detected as other local or distant metastasis. After that, wide excision of the skin lesion was performed. Although skin cancer is not commonly observed, it is necessary to rule out this disease entity by performing biopsy of skin lesions.
\end{abstract}

Keywords: Basal cell carcinoma / Fistula / Skin neoplasms

\section{INTRODUCTION}

Odontogenic cutaneous fistula is an uncommon disease and may be easily misdiagnosed. It occurs because of chronic dental draining infections, especially apical periodontitis [1]. The most common cause is endodontic or periodontal infections, trauma, retained roots and residual chronic infection of the jaws [2]. The location for extra oral sinus tracts are mostly the mandibular angles, chin and cheeks [3]. The chronic infections around apex of a dental root can drain to the mouth or less commonly to the skin via a sinus tract. Moreover, the draining sinus tracts can be located at a distance from the origin of infection [4]. Dental symptom like toothache is the most common symptom of odontogenic cutaneous fistula. However, only $50 \%$ of the patients experienced dental pain and the in-

\footnotetext{
Correspondence: Kyung Suk Lee

Department of Plastic and Reconstructive Surgery, Gyeongsang National University Hospital, 79 Gangnam-ro, Jinju 52727, Korea

E-mail: opensound@hanmail.net

Received January 2, 2017 / Revised May 30, 2017 / Accepted May 31, 2017
}

volved teeth are not always tender to percussion [5]. Therefore they could be easily initially misdiagnosed as skin lesions. The treatment of any odontogenic fistula lasting for more than three weeks should be surgically closed to avoid further medial problems and antibiotic helps to reduce infection in such a high risk situation [6]. Elimination of dental infection through endodontic treatments or tooth extraction is vital for the management of cutaneous sinus tracts [6]. This case report presents a basal cell carcinoma (BCC) that was diagnosed odontogenic cutaneous sinus tract.

\section{CASE REPORT}

A 64-year-old male patient had symptoms such as toothache and loose tooth \#13 and \#14 about one month ago. The dental pain was intermittent, and he did not go to the local hospital and took painkillers. Back then, there was no apparent skin problem at the right side of the patient's right nasal area. His pain began 3 weeks before the visit to the hospital. At that time, the pus spontaneously 
occurred in the right alar groove area and upper alveolar area (Fig. 1). After seeing the dentist, he scanned the computed tomography scan. Findings of abscess infiltration in the upper right alveolar region were observed on computed tomography (Fig. 2). He was di-

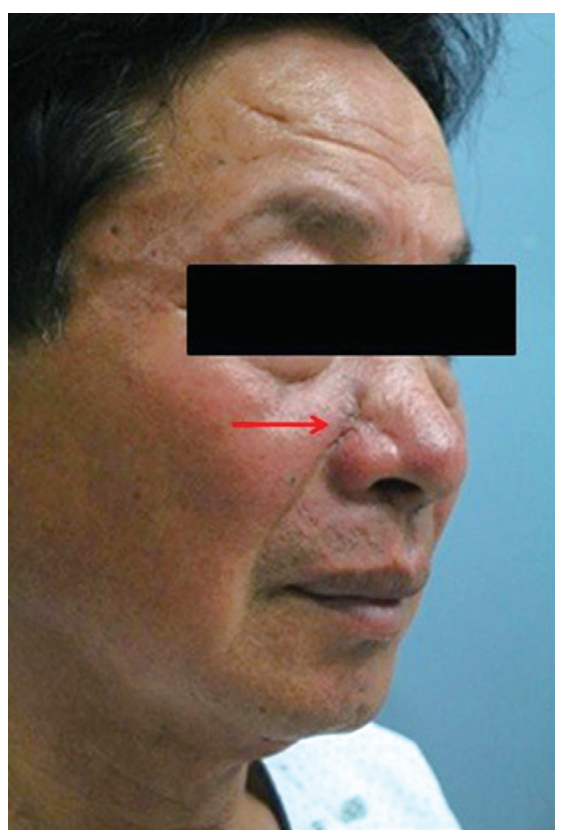

Fig. 1. A photograph taken a week after a dental surgery. The red arrow shows surrounding skin lesions with crusting and induration on the right alar groove area. agnosed with odontogenic fistula and performed treatments like debridement, tooth extraction, and fistulectomy with biopsy at a dental department. The biopsy results revealed basal cell carcinoma and inflamed granulation tissue with fistula. There was no clear correlation between fistula and skin cancer of the pathologic findings (Fig. 3). However, there was a doubt as to basal cell carcinoma which is close to fistula. The positron emission tomographycomputed tomography scan was checked to confirm the metastasis of cancer. The hypermetabolic lesions (standardized uptake value max values 5.2) were observed in right paranasal skin and subcutaneous layer. There were no significant fluorodeoxyglucose uptake lesions including surrounding lymph nodes. For the wound in right nasal alar area, wide excision including $0.5 \mathrm{~cm}$ sized safety margin was performed. The frozen section biopsy confirmed that there was no remaining tumor tissue, and primary closure was performed. He took antibiotics for about a week after the surgery. At the 6- month follow-up, the wound site had healed without any other problem.

\section{DISCUSSION}

We introduced a case of the basal cell carcinoma of the skin near-

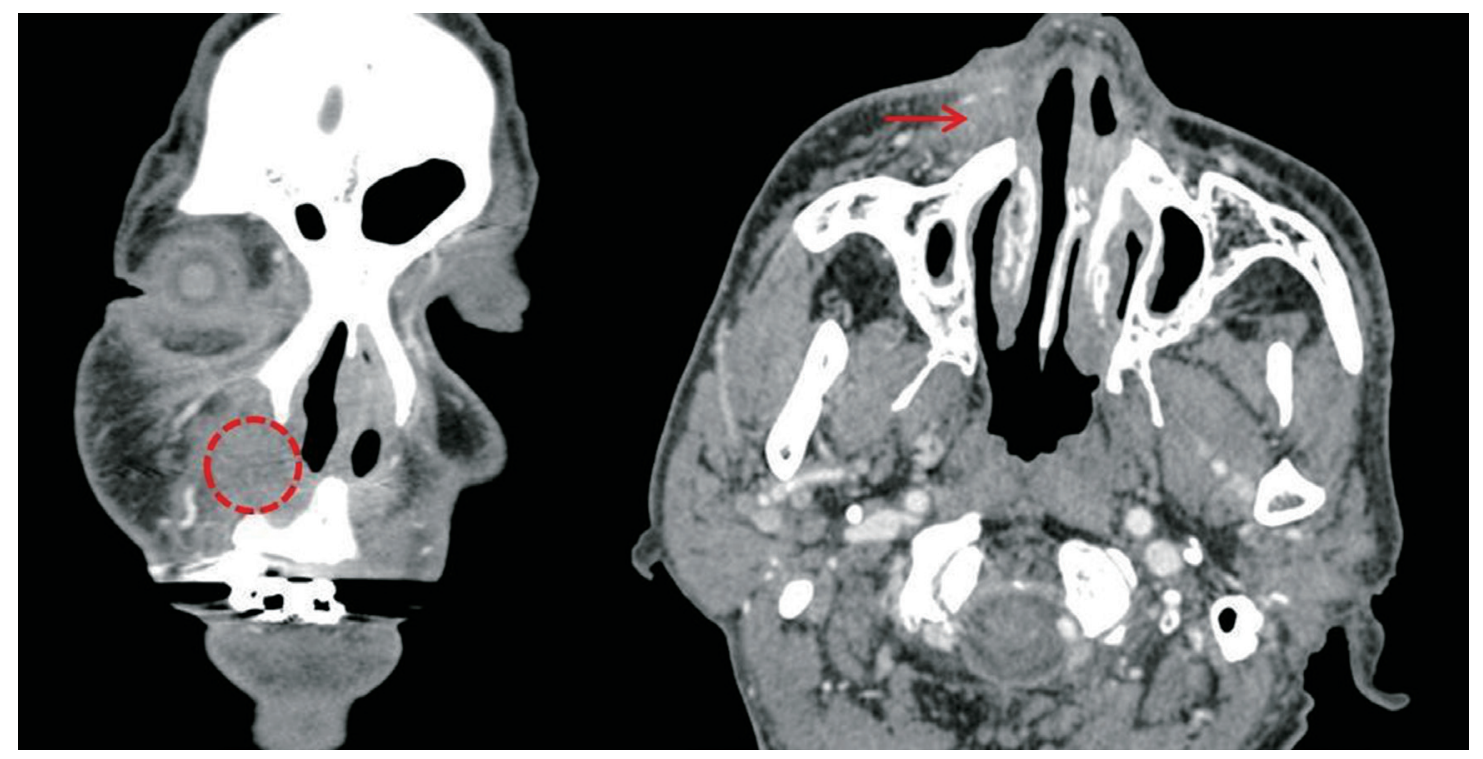

Fig. 2. Preoperative images (3D orbit enhanced computed tomography). On the left image, the red circle indicates the abscess infiltration around the root of right upper lateral incisor. On the right image, red arrow indicates the abscess infiltration under the indurated skin. 

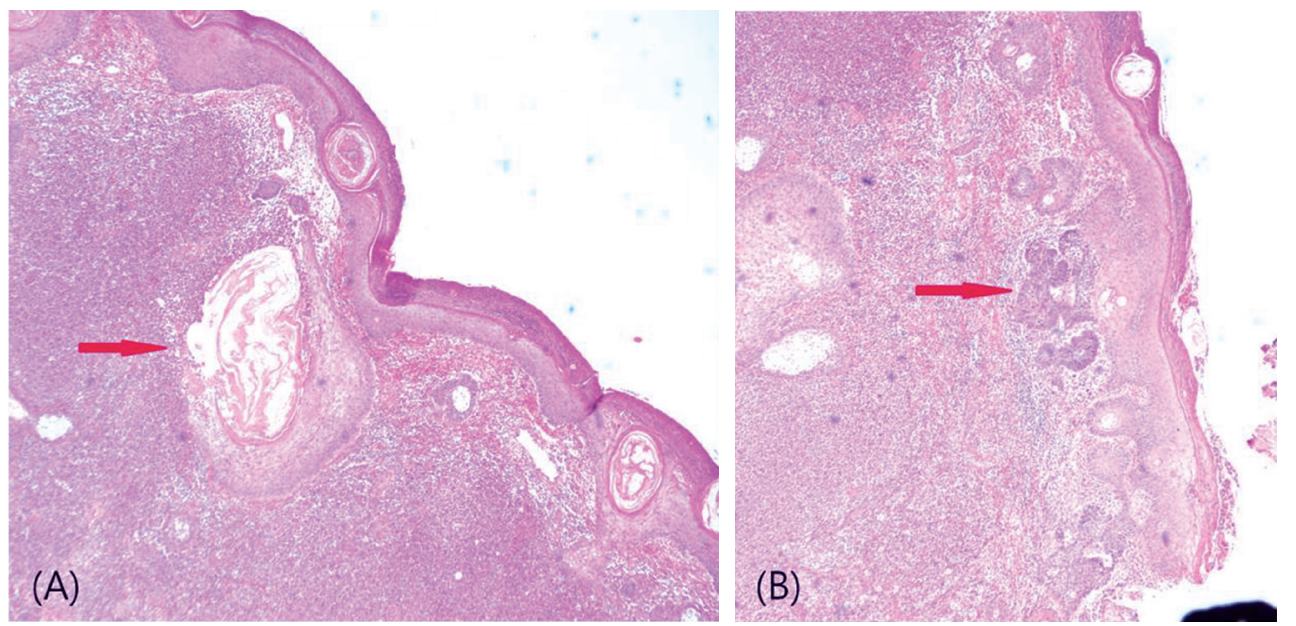

Fig. 3. The result of histologic findings showing a fistula (A) and a small islet of basaloid tumor cells, superficial basal cell carcinoma (B) at red arrows. $\mathrm{H} \& \mathrm{E}, \times 40$ (A); $\mathrm{H} \& \mathrm{E}, \times 40$ (B).

by odontogenic fistula. Dental pathogen is the most common cause to form cutaneous sinus track [1,2]. Patients often to find dermatologist because of only skin lesions. About $50 \%$ of these patients will experience unnecessary surgery and antibiotic treatment until a proper diagnosis is made [3-5]. Therefore, the importance of accurate diagnosis is emphasized with the physical examination and appropriate test. According to the literature for the odontogenic fistula treatment, one study reported that most patients can be healed within about 1-2 weeks without the particular treatment for the skin lesion caused by odontogenic fistula if a primary cause, especially infection is removed [6]. Studies using sections which can accurately detect basal cell carcinoma at any part of the surgical margin suggest that excision of small $(<20 \mathrm{~mm})$ well-defined lesions with a 3-mm peripheral surgical margin will clear the tumor in $85 \%$ of cases. A 4-5-mm peripheral margin will increase the peripheral clearance rate to approximately $95 \%$, indicating that approximately $5 \%$ of well-defined basal cell carcinomas extend over $4 \mathrm{~mm}$ beyond their apparent clinical margins. Surgical excision is a highly effective treatment for primary basal cell carcinoma, with a recurrence rate of $<2 \%$ reported 5 years following histologically complete excision [7]. However in most cases, the therapy such as dental and periodontal extraction, pus drainage, and fistulectomy etc. are needed because of the high possibilities of the recurrence with the common treatment of only skin lesion. Cohen and Eliezri [8] in 1990 have reported that cutaneous odon- togenic sinus was diagnosed after being misdiagnosed with basal cell carcinoma on right nasolabial fold.

We report on the case with a review of literature. We have experienced a rare case that is hardly found in the literature, which was diagnosed as basal cell carcinoma and odontogenic fistula in the patient's facial area clinically and pathophysiology. Although there was no clear evidence about the relation with basal cell carcinoma and odontogenic fistula, we believe that there is a possibility of a skin tumor caused by chronic inflammation from odontogenic fistula. Therefore, when odontogenic fistula was found, it would be advisable to check the skin cancer through the biopsy. If the skin cancer was confirmed, we should have a wide excision of it with safety margin. When there are skin lesions on the cheek and jaw area, the physical examination of skin lesion as well as oral cavity is also important. And also regular observation is required to check the local recurrence and distance metastasis of cancer after surgery. Although it is not common case, we have to keep in mind the possibility of skin cancer with odontogenic cutaneous fistula. Furthermore it is necessary to rule out the skin cancer through the biopsy for skin lesions.

\section{REFERENCES}

1. Tian J, Liang G, Qi W, Jiang H. Odontogenic cutaneous sinus tract associated with a mandibular second molar having a rare distolingual 
root: a case report. Head Face Med 2015;11:13.

2. Alasseri NA, Assari AS. Facial fistula. Long-term sequelae of a complicated exodontia. Saudi Med J 2015;36:490-3.

3. Samir N, Al-Mahrezi A, Al-Sudairy S. Odontogenic cutaneous fistula: report of two cases. Sultan Qaboos Univ Med J 2011;11:115-8.

4. Cantatore JL, Klein PA, Lieblich LM. Cutaneous dental sinus tract, a common misdiagnosis: a case report and review of the literature. $\mathrm{Cu}-$ tis 2002;70:264-7.

5. Rudagi KB, Rudagi BM. Cutaneous sinus tract of odontogenic origin a misdiagnosed lesion: report of two cases. Acta Stomatol Croat 2012;46:317-22

6. Borgonovo AE, Berardinelli FV, Favale M, Maiorana C. Surgical options in oroantral fistula treatment. Open Dent J 2012;6:94-8.

7. Telfer NR, Colver GB, Morton CA. Guidelines for the management of basal cell carcinoma. Br J Dermatol 2008;159:35-48.

8. Cohen PR, Eliezri YD. Cutaneous odontogenic sinus simulating a basal cell carcinoma: case report and literature review. Plast Reconstr Surg 1990;86:123-7. 